\title{
Towards Autonomous and Reliable Applications of UAVs and Remote Sensing Techniques for Forest Fire and Environment Surveillance
}

\author{
Youmin Zhang \\ Department of Mechanical, Industrial and Aerospace Engineering \\ Concordia Institute of Aerospace Design and Innovation (CIADI) \\ Concordia University \\ Montreal, Quebec, H3G 1M8, Canada \\ Ymzhang@encs.concordia.ca; Youmin.zhang@concordia.ca
}

Benefited from technical advances in materials, mechatronics, communication, computation, control, sensors, actuators and new/smart designs, Unmanned Aerial Vehicles (UAVs) are gaining more and more attention and rapid development during the last a few years due to their relatively easy and cost-effective uses in various application tasks such as surveillance, sensing, search and rescue, agriculture, forest, environment, pipelines, powerlines, military and security applications. In this keynote speech, brief overall view on the challenges and latest developments on Guidance, Navigation, and Control (GNC) of UAVs integrating with Remote Sensing (RS) techniques for autonomous, efficient and reliable applications to forest, powerline and environment monitoring and fires/damages/risks detection will be presented first, then some of new developments and current research works being carried out at presenter's group will be introduced as the second part of the presentation. In particular, new developments on Fault Detection and Diagnosis (FDD), Fault-Tolerant Control (FTC), FaultTolerant Cooperative Control (FTCC) techniques towards autonomous and reliable applications to the above-mentioned tasks, and the new technical developments for efficient and reliable detection of fires/damages/risks based on remotely sensed signals/images from onboard UAVs will be presented. 